\title{
Nucleotide composition of the Zika virus RNA genome and its codon usage
}

\author{
Formijn van Hemert and Ben Berkhout ${ }^{*}$
}

\begin{abstract}
Background: RNA viruses have genomes with a distinct nucleotide composition and codon usage. We present the global characteristics of the RNA genome of Zika virus (ZIKV), an emerging pathogen within the Flavivirus genus. ZIKV was first isolated in 1947 in Uganda, caused a widespread epidemic in South and Central America and the Caribbean in 2015 and has recently been associated with microcephaly in newborns.

Methods: The nearly $11 \mathrm{~kb}$ positive-stranded RNA genome of ZIKV was analyzed for its nucleotide composition, also in the context of the folded RNA molecule. Nucleotide trends were investigated along the genome length by skew analyses and we analyzed the codons used for translation of the ZIKV proteins.

Results: ZIKV RNA has a biased nucleotide composition in being purine-rich and pyrimidine-poor. This preference for purines is a general characteristic of the mosquito-borne and tick-borne flaviviruses. The virus-specific nucleotide bias is further enriched in the unpaired, single-stranded regions of the structured ZIKV RNA genome, thus further imposing this ZIKV-specific signature. The codons used for translation of the ZIKV proteins is also unusual, but we show that it is the underlying bias in nucleotide composition of the viral RNA that largely dictates these codon preferences.

Conclusions: The ZIKV RNA genome has a biased nucleotide composition that dictates the codon usage of this flavivirus. We discuss the evolutionary scenarios and molecular mechanisms that may be responsible for these distinctive ZIKV RNA genome features.
\end{abstract}

Keywords: Zika virus, ZIKV, Viral RNA genome, Nucleotide composition, Nucleotide bias, Purine-rich, Codon usage, RNA structure, Flavivirus

\section{Background}

Several members of the Flavivirus genus are the causative agents of significant diseases in humans, livestock and wildlife. These include dengue virus that affects more than 50 million people worldwide each year, West Nile virus and Japanese encephalitis virus that caused outbreaks in North America and Asia, respectively [1]. Zika virus (ZIKV) is an emerging pathogen in the family Flaviviridae that was first isolated in 1947 from a sentinel rhesus monkey placed in the Zika Forest near Lake Victoria in Uganda [2]. ZIKV is transmitted by mosquitoes, especially Aedes africanus, but the virus was also isolated from other Aedes species (reviewed in [3]).

\footnotetext{
* Correspondence: b.berkhout@amc.uva.nl

Laboratory of Experimental Virology, Department of Medical Microbiology, Center for Infection and Immunity Amsterdam (CINIMA), Academic Medical Center, University of Amsterdam, Meibergdreef 15, 1105 AZ Amsterdam, The Netherlands
}

(c) 2016 The Author(s). Open Access This article is distributed under the terms of the Creative Commons Attribution 4.0 International License (http://creativecommons.org/licenses/by/4.0/), which permits unrestricted use, distribution, and reproduction in any medium, provided you give appropriate credit to the original author(s) and the source, provide a link to the Creative Commons license, and indicate if changes were made. The Creative Commons Public Domain Dedication waiver (http://creativecommons.org/publicdomain/zero/1.0/) applies to the data made available in this article, unless otherwise stated.
Z4] causing a febrile illness with dengue fever like symptoms [5]. Sporadic cases were reported in sub-Saharan Africa and Southeast Asia, followed by an outbreak in Micronesia in 2007 and major epidemics that started around 2013 in New Caledonia, the Cook Islands, French Polynesia and Easter Island [6, 7]. A rather dramatic increase in the number of ZIKV cases was reported from the Americas starting in 2015, Brazil being the most affected country with around 1 million cases reported at the end of 2015 [8-10]. Here, also cases of neurological manifestations and the Guillain-Barré syndrome were described. Recent reports indicate a significant increase in the number of microcephaly cases among newborns in northeast Brazil, suggesting that ZIKV infection in pregnancy may trigger fetal malformations [11]. 
Neural progenitor cells can be infected by this virus, leading to attenuation of their growth [12].

Given the clinical relevance, we performed a detailed analysis of several features of the ZIKV RNA genome, including the nucleotide (nt) composition, also in the context of the structured RNA genome, and the viral codon usage. This insight can be central to the understanding of factors that govern virus evolution. Mutation pressure has been shown to be the dominant factor shaping the nucleotide composition and codon usage in mammalian genomes [13-15]. The ZIKV genome of almost $11,000 \mathrm{nts}$ encodes a single polyprotein of 3419 amino acids that is cleaved by the viral serine and cellular furin proteases into the functional domains: the Capsid (C), Precursor of membrane (prM), Envelope (E) and 7 non-structural proteins (NS) [2]. We report that the nucleotide composition of the ZIKV virus genome is strongly biased and this bias directly influences the codons used for translation of the viral proteins.

\section{Methods}

\section{ZIKV sequences}

Viral RNA genome sequences were obtained from GenBank. The MR-766 prototype ZIKV strain originates from the index case: a monkey infected in 1947 in Uganda (Genbank entry NC_012532). Other ZIKV isolates used: KU497555 (Brazil), KU509998 (Haiti), KU501215 (Puerto Rico), KU312312 (Suriname), KU 647676 (Martinique), KJ776791 (French Polynesia), KU701217 (Guatamala), KU681082 (Philippines) and KF268950 (Central African Republic). The full genome sequences were manually curated into bona fide open reading frames.

\section{Maximum Likelihood (ML) phylogenetic analysis}

Phylogenetic and molecular evolutionary analyses were conducted with MEGA v6 [16]. The open reading frames (ORFs) of the different ZIKV strains were translated into amino acid sequences, which were aligned by means of the MUSCLE tool. The JTT $+\mathrm{G}$ model for assessing amino acid replacements during ZIKV evolution turned out to be the best fitting model judged by BIC score (Bayesian Information Criterion, 22469.52863) and AICc value (corrected Akaike Information Criterion, 22317. 61634). Non-uniformity of evolutionary rates among sites was modeled by a discrete Gamma distribution (+G $=0.554328239)$ with 5 rate categories. The ML value for model selection was $\log \mathrm{L}=-11140.79817$. All sites were used for phylogenetic analysis. A bootstrap test (1000 replicates) indicated robustness of the analysis. The hypothetical ancestral sequence (MRCA, Most Recent Common Ancestor) was constructed by means of the FastML server with the advanced options activated
[17]. The MLtree was rooted on the MRCA branch to show the evolutionary course of events.

\section{RNA structure prediction}

RNA secondary structure prediction was performed by the MFold v3.6 algorithm with default settings [18]. The MFold output file provided the ss-count, a frequency value that indicates whether an individual nucleotide (nt) is unpaired in the collection of folded structures (maximally 50). We scored an unpaired nt (singlestranded, "ss") if present in at least half of the RNA structures. Nts with a lower ss-count were scored double-stranded ("ds"). Excel was used for ss/ds discrimination and we generated fasta files to determine the nucleotide composition in MEGA v6 [16]. Because the size limit for submission to the MFold server is $9000 \mathrm{nts}$, the ZIKV RNA genome was partitioned into two fragments with 1000 nts overlap. We arithmetically averaged the ss-count data in the overlap before ss/ds discrimination was performed.

\section{Skew analysis}

Base composition along the complete RNA genome length and the accompanying ss and ds fasta files was analyzed by cumulative skew diagrams using overlapping windows $[19,20]$. Overlapping windows were defined around $1 \%$ of the sequence length with a step size of $20 \%$ of the window size, which generated about 500 data points per analysis irrespective of sequence length. A skew between nts $\mathrm{N} 1$ and N2 is defined as (N1 - N2)/ $(\mathrm{N} 1+\mathrm{N} 2)$. A positive value indicates that $\mathrm{N} 1$ exceeds $\mathrm{N} 2$.

\section{Codon usage}

The single ZIKV reading frame was analyzed using the "Nc-plot", which plots the effective number of codons (ENC-values) versus the GC-content at the $3^{\text {rd }}$ codon position (GC3) [21]. A continuous line indicates ENC values expected (ENCexp) for random codon usage at that particular GC3 value. Deviation from this line in the direction of lower ENC-values (observed ENC values, ENCobs) points to the selection of a preferred set of codons as described for highly expressed genes in yeast [22] and Escherichia coli [23]. The ratio ENCobs/ENCexp provides an easy measure of this deviation. A ratio value of 1 indicates zero codon bias. Values close to 1 (0.8 to 1.0$)$ indicate very weak or virtually absent codon bias. ENC and GC3 values of sequence data were determined by means of Simmonic 2005 v1.5 software [24]. ENC and GC3 values human and Aedes genes were derived from codon usage tables [25]. All calculations were performed in Excel v14.0.7128.5000. 


\section{Results}

Nucleotide composition of the ZIKV RNA

We first analyzed the complete RNA genome of the ZIKV MR-766 prototype strain [2], which originates from a sentinel monkey that became infected in Uganda in 1947 (Genbank entry NC_012532). This positivestranded RNA genome contains a single extended open reading frame (ORF) that encodes the viral polyprotein, which is subsequently processed into the different structural and enzymatic components. The ZIKV RNA genome is 10794 nucleotides (nts) long, with a short 5'untranslated region (UTR) of 106 nts and a 3'-UTR of 428 nts. The four possible nucleotides are not used at equal frequencies, the genome composition ranges from $2305 \mathrm{U}(21.3 \%)$ to $3139 \mathrm{G}$ (29.1\%). Table 1 summarizes these numbers and provides some further details. Overall, the RNA genome is enriched for purines $(\mathrm{G}+\mathrm{A}$, $56.8 \%$ ) over pyrimidines $(\mathrm{U}+\mathrm{C}, 43.2 \%)$, yielding a $\mathrm{Pu} /$ Py ratio of 1.31 . We will relate these ZIKV properties to that of other flaviviruses in the discussion.

These basic characteristics do not deviate significantly for other ZIKV isolates. We analyzed two early isolates (Uganda and Central African Republic) and 8 recent isolates. Phylogeny of these 10 strains is presented in Fig. 1, which is in agreement with published data [11, 26, 27]. ZIKV from the Philippines is ancestral to those constituting the recent epidemic in southern America. The root of the trifurcated tree indicates that a putative most recent common ancestor (MRCA) circulated in Africa approximately 91 years ago, assuming a constant virus evolution rate and an age of 68 years for the prototype Uganda virus. The typical nucleotide composition and purine preference are common features among the ancient and more recent taxa of this ZIKV collection. For instance, the recently isolated Natal RGN strain isolated from a Brazilian child with microcephaly [11] contains $21.4 \% \mathrm{U}$ and $29.2 \% \mathrm{G}$, with a preference for purines of $56.6 \%$. We conclude that these nt characteristics are constant properties among ZIKV variants, at least those circulating in the last century.

This nt bias is likely to influence the codon usage in the single ORF that encodes the ZIKV polyprotein, but in fact the same nt trends are apparent for segments of the RNA genome that are not translated into protein: the short 5'-untranslated region (5'UTR) and 3'UTR of the ZIKV genome (Table 1). These regions are very short, 106 and 428 nts respectively for the MR-766 prototype genome, making a statistically sound analysis of the nt-count precarious, but when combined these domains exhibit exactly the same nt ranking order as the full-length RNA: G $(29.0 \%)>$ A $(26.6 \%)>$ C $(25.1 \%)>$ U (19.3\%). Local fluctuations may occur especially near the 5' and 3' termini of the genome due to the presence of essential sequence elements that are involved in viral genome replication. For instance, the U-count of the 5'UTR is elevated, in part due to the presence of four U3 stretches in this 106-nt segment. Conservation of these specific molecular signals among different ZIKV strains argues for such a biological role, e.g. the 107nts 5'UTR of the Natal RGN isolate encodes three U3 and two U4 stretches. In addition, the genome ends may encode specific RNA structures with a replicative function [2].

\section{Composition of the structured RNA genome}

We previously reported that the unequal nucleotide composition of a viral RNA genome may become even more biased in unpaired domains of the folded RNA molecule. This initial study was performed for HIV-1 RNA, using both MFold-generated structure prediction and the experimentally probed RNA structure [28, 29]. The structure of the ZIKV RNA genome was predicted by MFold. We investigated maximally 50 folded structures to provide each individual nt a frequency value of being unpaired (single-stranded, ss) in this collection of predicted structures. We subsequently counted the number of ss and ds (double-stranded, paired) positions (Table 2). Paired positions are in excess over unpaired nucleotides in these folded RNA genomes, with a dsRNA value of $62.0 \%$. One major and several minor trends can be recognized. First and most importantly, we observed a strong A-accumulation for the unpaired ss segments of the ZIKV RNA genome (from 27.7 to $44.7 \%$ ), which equally means A-depletion (from 27.7 to $17.2 \%)$ in the paired ds genome domains. All non-A nts seem to compensate for these strong A-movements. In other words, G, U and C increase their prevalence in the paired domains, but lose incidence in the unpaired domains due to the prominent A-pressure. The unpaired part of ZIKV RNA is particularly A-rich (44.7 \%) and Cpoor $(14.6 \%)$, with a multitude of purines $(G+A$, $68.3 \%)$. These properties may be relevant for interaction

Table 1 ZIKV RNA composition

\begin{tabular}{|c|c|c|c|c|c|c|}
\hline ZIKV RNA & nts & $A$ & $U$ & $\mathrm{C}$ & G & $\mathrm{Pu} / \mathrm{Py}$ \\
\hline complete & 10794 & 2991 (27.7 \%) & 2305 (21.3\%) & 2359 (21.9\%) & 3139 (29.1\%) & 1.31 \\
\hline $5^{\prime} U T R$ & 106 & 29 & 31 & 16 & 30 & \\
\hline 3'UTR & 428 & 113 & 72 & 118 & 125 & \\
\hline $5^{\prime}+3^{\prime} U T R$ & 534 & $142(26.6 \%)$ & 103 (19.3\%) & 134 (25.1 \%) & 155 (29.0 \%) & 1.25 \\
\hline
\end{tabular}




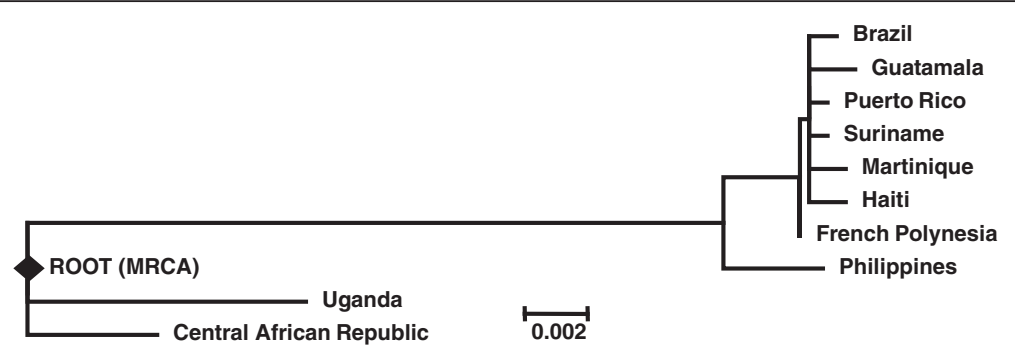

Fig. 1 Molecular phylogeny of ZIKV strains. The polyprotein ORFs were translated into the amino acid sequence and a hypothetical ancestral sequence, generated with FastML, was added to the alignment. The Most Recent Common Ancestor (MRCA) was predicted to have been around 91 years ago, see the results section for further details. We performed 1000 bootstrap replicates that support the robustness of the phylogenetic analysis. The Log Likelihood value $(\mathrm{LnL})$ of tree building amounted to -11055.85 . The scale bar indicates the number of amino acid substitutions per site

with intracellular sensors of the innate immune systems in humans and Aedes [30-32]. A more definitive analyses should be performed when the experimentally probed structure of the ZIKV virus RNA genome becomes available.

\section{Genome skew analysis}

A powerful way to visualize trends in nt usage is a nucleotide skew analysis along the viral genome (Fig. 2). The general trends presented above were confirmed. Note that GA in skew language does not represent a base pair, but rather a comparison of the number of $G$ with the number of A nts. The skew lines are generally straight, indicating that the observed trends are steady along the $11 \mathrm{~kb}$ genome, without significant positional effects along the viral genome, e.g. in the 5' and 3'UTRs versus the extended ORF. The skew analysis of the complete genome is presented in the left panel (all nts). This assessment underscores the preference of purines over pyrimidines as any direct $\mathrm{Py}-\mathrm{Pu}$ comparison is won by the latter, resulting in a declining line for CA, UA, CG and UG. Only the Pu-Pu (GA) and Py-Py (UC) comparisons showed a lack of favorite nt.

We subsequently analyzed the ds and ss positions separately (Fig. 2, ds in middle panel, ss in right panel). The skew lines show more divergence in both the ds and ss segments compared with the all-nt skew analysis. It is also immediately apparent that the ds and ss positions act as communicating vessels. The gain of the A-count in the ss segment (strongly declining lines for GA, UA and especially CA) is mirrored by a loss in the ds compartment (strongly rising line for UA, CA and
GA). G seems the second best option in the ss compartment (declining line for UG and CG). In fact, these trends do not mimic any of the virus-specific trends that we described previously, e.g. for retroviruses and coronaviruses [20, 33-35].

\section{Codon analysis}

It is quite usual to perform an autonomous codon analysis, but we purposely first presented the general genome characteristics as these may largely influence the codon usage. It is immediately clear that ZIKV does not use all codons at equal frequency, but the patterns seem to vary (Table 3). Among the 4-codon groups (Ala, Gly, Pro, Thr and Val), but also the 4-codon set within the 6codon groups (Leu, Arg and Ser), two quite opposite patterns can be recognized. Either the A-ending codons prevail (Pro, Ser, Thr), or the G-ending codons win (Leu, Val). Some outliers are also apparent: the Gly 4-codon set prefers both $A$ and $G$, the Ala 4-codon set prefers $C$ with $A$ as second best, and the 4-codon set within the 6codon group Arg has $\mathrm{C}$ as the best option, but with $\mathrm{G}$ as the second best. The opposing $\mathrm{A} / \mathrm{G}$ trend is even more remarkable. When A wins, $G$ does not become the second-best, but always drops to the lowest value. Inspection of the codons provides a possible explanation for this restriction as the suppressed codons encode a CpG motif (marked in bold and by underlining in Table 3). The CpG motif is a methylation signal in DNA, but is also recognized by host innate immune sensors as a pathogen signature [36], and is discriminated against in many RNA viruses, including poliovirus and the Flaviviridae [37, 38]. For ZIKV RNA, a striking $\mathrm{GpC} / \mathrm{CpG}$

Table 2 Nucleotide composition in ss/ds segments

\begin{tabular}{llllll}
\hline ZIKV RNA & nts & A & $U$ & C & G \\
\hline all & 10794 & $2991(27.7 \%)$ & $2305(21.3 \%)$ & $2359(21.9 \%)$ & $3139(29.1 \%)$ \\
ds & 6687 & $1154(17.2 \%)$ & $1604(24.0 \%)$ & $1758(26.3 \%)$ & $2171(32.5 \%)$ \\
SS & 4107 & $1837(44.7 \%)$ & $701(17.1 \%)$ & $601(14.6 \%)$ & $968(23.6 \%)$ \\
\hline
\end{tabular}




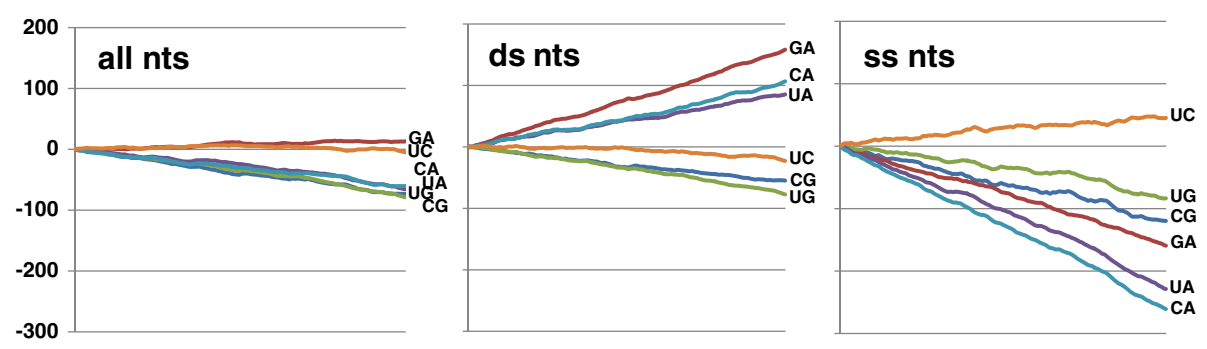

Fig. 2 Nucleotide skew analysis of the ZIKV RNA genome. Skew values (N1 - N2)/(N1 + N2) were calculated in overlapping windows along the sequence. Window size was set at $1 \%$ of the length of the sequence with a step size of $20 \%$ of the window size, resulting in approximately 500 data points comprising the $\mathrm{X}$-axis. We used the same $\mathrm{Y}$-axis for the cumulative skew values to allow a direct comparison of the compositional signatures of different genome parts: "all nts", "ds nts" and "ss nts"

ratio of 2.17 was scored (analysis not shown). In other words, it seems that $G$ wins unless a $C p G$ motif is created.

A second dinucleotide motif that is discriminated against is UpA with an ApU/UpA ratio of 1.86. This explains most cases where A-ending codons are losing (marked in bold and by underling in Table 3), e.g. within the Leu 6-codon set it clarifies the choices made for both the 2-codon and 4-codon sets. The complete dinucleotide analysis (not shown) indicates that the all purine (GpA/ApG) and all pyrimidine $(\mathrm{CpU} / \mathrm{UpC})$ choices are unbiased compared to the four purine/pyrimidine combinations (results not shown). Both CpG and UpA discrimination have been reported for other flaviviruses [39, 40], but surprisingly little attention was given to the purine/pyrimidine composition as the potential unifying signature.

Overall, the purines $\mathrm{G}$ or A seem to dominate the codon choices made, and U/C choices seem relatively balanced. The G-bias is also apparent in the 2-codon groups (G/A column in Table 3), where $G$ wins over A in 3 of 5 case, with a draw for the Gln group and a unique A-win for the 2-codon set within the outlier Arg 6-codon group. Only modest effects were scored for the $\mathrm{U} / \mathrm{C}$ choice in the 2-codon groups, but $\mathrm{C}$ wins in 4 out of 7 cases with two draws, consistent with the overall nt count. Thus, codon usage in ZIKV RNA seems to follow the nt compositional trend with regard to the purine/ pyrimidine bias that is present across the viral genome.

Codon usage in the single ZIKV ORF was also analyzed by means of an "Nc-plot" (Fig. 3), with the effective number of codons (ENC-values) plotted versus the GCcontent at the $3^{\text {rd }}$ codon positions (GC3). The continuous grey line indicates the ENC values expected (ENCexp) for random codon usage at a particular GC3 value. Deviation from this line in the direction of lower ENC-values (observed ENC values, ENCobs) points to the selection of a preferred set of codons. The ratio ENCobs/ENCexp provides an easy measure for the extent of deviation: the value of 1 (ENCobs $=$ ENCexp) indicates the absence of any codon bias, values below 0.8 may suggest weak codon bias, and even lower values can advocate stronger codon bias. We included two early and several recent ZIKV isolates, but all cluster in a very tiny area of the Nc-plot, confirming their very close genetic relationship. Most importantly, the ENCobs/ ENCexp ratio of all ZIKV isolates is above 0.88, which is close to the unbiased value. We also plotted ENC/GC3 values derived from average codon usage in a set of human and mosquito mRNAs (Homo and Aedes in Fig. 3). ZIKV and human ENC/GC3 values are very similar. The mean GC3 value for Aedes genes is somewhat higher (0.63) compared with the values for ZKIV and human genes (0.53). Between virus and hosts we did not observe different codon preferences, which are related to codon bias by translational control and indicated by low ENCobs/ENCexp values. Overall, this analysis suggests that the virus-specific codon usage trends reflect the biased nt composition of these viral RNA genomes.

\section{Discussion}

This survey of the ZIKV RNA genome indicates a preference for purines over pyrimidines that is enhanced in the unpaired domains of the viral RNA and that influences the codons used for translation of the viral proteins. No significant differences were found among the highly related ZIKV strains concerning these basic properties, ranging from the prototype Uganda strain isolated in 1947 to recent south American isolates. The strong correlation between nucleotide composition and codon usage bias suggests that mutation pressure in ZIKV is an important determinant of the codon bias observed. This finding is consistent with previous findings for other viruses, which demonstrate a wide variation in codon usage that usually correlates with the viral RNA-specific nucleotide composition [41]. The ZIKV nt characteristics are quite distinctive from those of other viruses and may thus help to comprehend virus evolution and to provide an additional tool for virus classification purposes or the development of diagnostic reagents for 
Table 3 ZIKV codon usage

\begin{tabular}{|c|c|c|c|c|c|c|c|c|c|c|c|c|c|c|c|c|}
\hline$\overline{\mathrm{AA}}$ & Codon $^{a}$ & Number & Proportion & 4-group ${ }^{a}$ & 6-group ${ }^{1 a}$ & $\mathrm{G} / \mathrm{A}$ & $U / C$ & | & AA & Codon $^{\mathrm{a}}$ & Number & Proportion & 4-group ${ }^{a}$ & 6-group ${ }^{a}$ & $\mathrm{G} / \mathrm{A}$ & $\mathrm{U} / \mathrm{C}$ \\
\hline \multirow[t]{4}{*}{ Ala } & GCG & 30 & 0.11 & $\begin{array}{l}C>A> \\
U>\underline{G}\end{array}$ & & & & I & Asn & AAU & 36 & 0.35 & & & & $\begin{array}{l}C> \\
U\end{array}$ \\
\hline & GCA & 80 & 0.28 & & & & & | & & AAC & 68 & 0.65 & & & & \\
\hline & GCU & 77 & 0.27 & & & & & | & Pro & CCG & 11 & 0.08 & $\begin{array}{l}A>C> \\
U>\mathbb{G}\end{array}$ & & & \\
\hline & GCC & 96 & 0.34 & & & & & | & & CCA & 65 & 0.46 & & & & \\
\hline \multirow[t]{2}{*}{ Cys } & UGU & 35 & 0.55 & & & & $\begin{array}{l}U> \\
C\end{array}$ & | & & $\mathrm{CCU}$ & 28 & 0.2 & & & & \\
\hline & UGC & 29 & 0.45 & & & & & | & & $\mathrm{CCC}$ & 38 & 0.27 & & & & \\
\hline \multirow[t]{2}{*}{ Asp } & GAU & 61 & 0.4 & & & & $\begin{array}{l}C> \\
U\end{array}$ & | & Gln & CAG & 38 & 0.5 & & & $\begin{array}{l}G= \\
A\end{array}$ & \\
\hline & GAC & 93 & 0.6 & & & & & | & & CAA & 38 & 0.5 & & & & \\
\hline \multirow[t]{2}{*}{ Glu } & GAG & 115 & 0.52 & & & $\begin{array}{l}G> \\
A\end{array}$ & & | & Arg & AGG & 57 & 0.26 & & $A>G$ & & \\
\hline & GAA & 107 & 0.48 & & & & & | & & AGA & 93 & 0.42 & & & & \\
\hline \multirow[t]{2}{*}{ Phe } & UUU & 45 & 0.49 & & & & $\begin{array}{l}C= \\
U\end{array}$ & | & & CGG & 21 & 0.1 & & $\begin{array}{l}C>G> \\
U>A\end{array}$ & & \\
\hline & UUC & 46 & 0.51 & & & & & | & & CGA & 11 & 0.05 & & & & \\
\hline \multirow[t]{4}{*}{ Gly } & GGG & 67 & 0.22 & $\begin{array}{l}A>G> \\
C>U\end{array}$ & & & & | & & CGU & 14 & 0.06 & & & & \\
\hline & GGA & 150 & 0.49 & & & & & | & & CGC & 24 & 0.11 & & & & \\
\hline & GGT & 40 & 0.13 & & & & & | & Ser & AGU & 36 & 0.18 & & $C=U$ & & \\
\hline & GGC & 51 & 0.17 & & & & & | & & AGC & 38 & 0.19 & & & & \\
\hline \multirow[t]{2}{*}{ His } & CAU & 34 & 0.46 & & & & $\begin{array}{l}C> \\
U\end{array}$ & | & & UCG & 18 & 0.09 & & $\begin{array}{l}A>U> \\
C>\mathbb{G}\end{array}$ & & \\
\hline & CAC & 40 & 0.54 & & & & & | & & UCA & 50 & 0.25 & & & & \\
\hline \multirow[t]{3}{*}{ Ile } & AUA & 51 & 0.29 & & & & & | & & UCU & 29 & 0.15 & & & & \\
\hline & $A \cup U$ & 46 & 0.26 & & & & & | & & UCC & 28 & 0.14 & & & & \\
\hline & $A \cup C$ & 78 & 0.45 & & & & & | & Thr & $\mathrm{ACG}$ & 20 & 0.09 & $\begin{array}{l}A>C> \\
U>\underline{G}\end{array}$ & & & \\
\hline \multirow[t]{2}{*}{ Lys } & AAG & 111 & 0.59 & & & $\begin{array}{l}G> \\
A\end{array}$ & & | & & $\mathrm{ACA}$ & 91 & 0.4 & & & & \\
\hline & AAA & 77 & 0.41 & & & & & | & & $A C U$ & 53 & 0.23 & & & & \\
\hline \multirow[t]{6}{*}{ Leu } & UUG & 69 & 0.22 & & $G>\underline{A}$ & & & | & & ACC & 62 & 0.27 & & & & \\
\hline & $\underline{U A A}$ & 20 & 0.06 & & & & & | & Val & GUG & 115 & 0.42 & $\begin{array}{l}G>C> \\
U>\underline{A}\end{array}$ & & & \\
\hline & CUG & 94 & 0.3 & & $\begin{array}{l}G>C> \\
U>\underline{A}\end{array}$ & & & | & & GUA & 31 & 0.11 & & & & \\
\hline & CUA & 31 & 0.1 & & & & & | & & GUU & 55 & 0.2 & & & & \\
\hline & CUU & 46 & 0.15 & & & & & | & & GUC & 70 & 0.26 & & & & \\
\hline & CUC & 53 & 0.17 & & & & & | & Trp & UGG & 92 & 1 & & & & \\
\hline \multirow[t]{2}{*}{ Met } & AUG & 130 & 1 & & & & & | & Tyr & UAU & 38 & 0.44 & & & & $\begin{array}{l}C> \\
U\end{array}$ \\
\hline & & & & & & & & | & & UAC & 49 & 0.56 & & & & \\
\hline
\end{tabular}

${ }^{\mathrm{a} C p G}$ and UpA in bold/underlined

improved surveillance of this class of emerging pathogens. A quick comparison of ZIKV to other members of the family Flaviviridae, including the major pathogens dengue virus [42], yellow fever virus, Japanese encephalitis virus, West Nile virus [43] and hepatitis C virus, indicated a similar purine-preference for the mosquito-borne and tick-borne flaviviruses and the pestivirus genus, but not for the hepacivirus group (results 


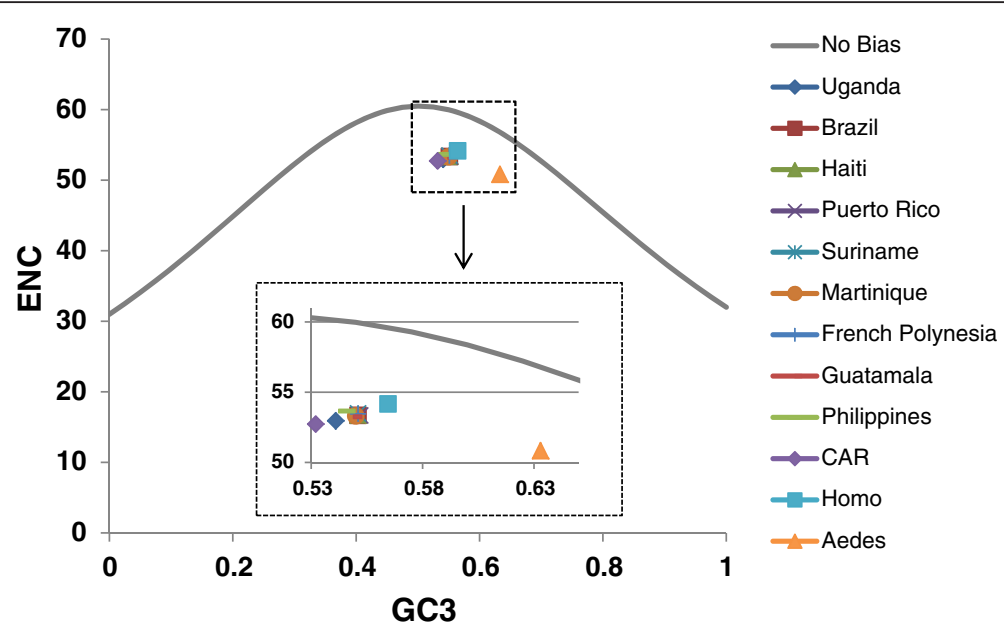

Fig. 3 Codon ENC analysis of ZIKV RNA. The effective number of codons (ENC-values, Y-axis) of the single ZIKV ORF (3420 codons) was plotted against the GC-content at the 3rd synonymous codon positions (GC3-values, $X$-axis). The continuous grey line represents theoretical ENC values for random codon usage as a function of GC3. Deviation from this line in the direction of lower ENC-values points to selection of a preferred set of codons. We analyzed codon usage for several ZIKV isolates and the two host species (homo sapiens and Aedes aegypti). The latter two values are based on the following numbers of codons (with the number of genes in parentheses, see [25]): human: 40,662,582 $(93,487)$ and Aedes aegypti: 257,935 (585)

not shown). Intriguingly, these purine-loving viruses seem to favor either A or G, similar to the "communicating" pyrimidine vessels described for coronaviruses [35]. Although quite extensive codon analyses have been conducted for flaviviruses [42-50], as far as we know the typical purine/pyrimidine pattern has not yet been described previously.

We previously presented two possible causes for the presence of viral RNA genomes with a biased ntcomposition [51]. One frequently entertained possibility is that this is due - over evolutionary times - to mutational bias of the viral polymerase [52]. Alternatively, we suggested a specific genome composition may be selected for a specific function, e.g. to facilitate RNA packaging in the virion particle or to prevent recognition by innate immune sensors in the infected cell. For HIV-1, there are recent indications to support both functional scenario's [30, 53]. For ZIKV, one cannot formally exclude that functions are executed by the viral minus-RNA strand, the critical replication intermediate that in terms of nt-composition is the mirror image of the viral plus-strand RNA genome. In addition, the double-stranded RNA replication intermediate and its nt-composition and structure may be screened by several innate immune sensors in the human, monkey and Aedes hosts.

We previously reported that virus-specific compositional signatures are commonly enhanced in the unpaired domains of a structured RNA genome. This is true for HIV-1, with an average A-count of $36.2 \%$ that increases to $47.5 \%$ in the unpaired genome segments, and also for other retroviruses with a distinct nt bias [28,
34]. We recently analyzed coronaviruses and reported some common characteristics (high $\mathrm{U}$, low $\mathrm{C}$ count), but also species-specific signatures that differentiate the pathogenic MERS/SARS strains from other coronaviruses [35]. Again, nucleotide biases were boosted in the unpaired domains of the viral RNA genome. The concentration of nt-bias in certain genome domains may have been selected for a certain function.

Many reports dwell on the exotic codon usage employed by viruses. For instance, a recent report documented codon usage adaptation in pandemic ZIKV virus strains [54]. However, a sobering finding is that this bias usually coincides with a bias in nt-composition of the viral RNA genome, and therefore does not represent translational selection of certain codons and/or the matching tRNA species. This also seems true for ZIKV, which preferentially uses A- or G-rich codons, but this seems to be a direct consequence of having a purinerich RNA genome (56.8 \%). HIV is the only virus in this collection that combines A-accumulation with weak translational selection by means of codon bias. Previously, we have even documented a tendency in HIV proteins for selection of amino acids encoded by Arich codons [55]. Codon usage can become an important aspect when it comes to optimization of protein production, e.g. in the context of ZIKV vaccine development where efficient viral gene expression may be required to generate immunity [56]. Given the biased codon usage of this virus, it is important to change towards synonymous codons that are more favored in the relevant production platform, e.g. human cell lines. 


\section{Acknowledgements}

We thank Alexander Pasternak and Tonja van der Kuyl for critical reading of the manuscript. RNA research in the BB laboratory is sponsored by NWOChemical Sciences (TOP grant).

\section{Authors' Contribution}

$\mathrm{BB}$ conceived the study. FvH and BB participated in the study design. $\mathrm{FvH}$ performed the analyses. BB wrote the manuscript and FvH helped to draft the manuscript and produced all Figures and Tables. Both authors read and approved the final manuscript.

\section{Competing interests}

The authors declare that they have no competing interests.

\section{Received: 1 March 2016 Accepted: 26 May 2016}

Published online: 08 June 2016

\section{References}

1. Kuno G, Chang GJ. Biological transmission of arboviruses: reexamination of and new insights into components, mechanisms, and unique traits as well as their evolutionary trends. Clin Microbiol Rev. 2005;18(4):608-37.

2. Kuno G, Chang GJ. Full-length sequencing and genomic characterization of Bagaza, Kedougou, and Zika viruses. Arch Virol. 2007;152(4):687-96.

3. Berthet N, Nakoune E, Kamgang B, Selekon B, Descorps-Declere S, Gessain A, Manuguerra JC, Kazanji M. Molecular characterization of three Zika flaviviruses obtained from sylvatic mosquitoes in the Central African Republic. Vector Borne Zoonotic Dis. 2014;14(12):862-5.

4. Simpson Dl. Zika Virus Infection in Man. Trans R Soc Trop Med Hyg. 1964:58:335-8

5. Simon F, Javelle E, Oliver M, Leparc-Goffart I, Marimoutou C. Chikungunya virus infection. Curr Infect Dis Rep. 2011;13(3):218-28

6. Duffy MR, Chen TH, Hancock WT, Powers AM, Kool JL, Lanciotti RS, Pretrick M, Marfel M, Holzbauer S, Dubray C, et al. Zika virus outbreak on Yap Island, Federated States of Micronesia. N Engl J Med. 2009;360(24):2536-43.

7. Cao-Lormeau VM, Roche C, Teissier A, Robin E, Berry AL, Mallet HP, Sall AA, Musso D. Zika virus, French polynesia, South pacific, 2013. Emerg Infect Dis. 2014;20(6):1085-6.

8. Bogoch II, Brady OJ, Kraemer MU, German M, Creatore MI, Kulkarni MA Brownstein JS, Mekaru SR, Hay SI, Groot E, et al. Anticipating the international spread of Zika virus from Brazil. Lancet. 2016;387(10016):335-6.

9. Calvet GA, Filippis AM, Mendonca MC, Sequeira PC, Siqueira AM, Veloso VG, Nogueira RM, Brasil P. First detection of autochthonous Zika virus transmission in a HIV-infected patient in Rio de Janeiro, Brazil. J Clin Virol. 2016;74:1-3.

10. Musso D. Zika virus transmission from French Polynesia to Brazil. Emerg Infect Dis. 2015;21(10):1887

11. Mlakar J, Korva M, Tul N, Popovic M, Poljsak-Prijatelj M, Mraz J, et al. Zika Virus Associated with Microcephaly. N Engl J Med. 2016.

12. Tang H, Hammack C, Ogden SC, Wen Z, Qian X, Li Y, et al. Zika Virus Infects Human Cortical Neural Progenitors and Attenuates Their Growth. Cell Stem Cell. 2016.

13. Sharp PM, Stenico M, Peden JF, Lloyd AT. Codon usage: mutational bias, translational selection, or both? Biochem Soc Trans. 1993;21(4):835-41.

14. Wolfe KH, Sharp PM, Li WH. Mutation rates differ among regions of the mammalian genome. Nature. 1989;337(6204):283-5.

15. Francino MP, Ochman $\mathrm{H}$. Isochores result from mutation not selection. Nature. 1999:400(6739):30-1.

16. Tamura K, Stecher G, Peterson D, Filipski A, Kumar S. MEGA6: Molecular Evolutionary Genetics Analysis version 6.0. Mol Biol Evol. 2013:30(12):2725-9.

17. Ashkenazy H, Penn O, Doron-Faigenboim A, Cohen O, Cannarozzi G, Zomer O, Pupko T. FastML: a web server for probabilistic reconstruction of ancestral sequences. Nucleic Acids Res. 2012;40(W1):W580-4.

18. Zuker M. Mfold web server for nucleic acid folding and hybridization prediction. Nucleic Acids Res. 2003;31(13):3406-15

19. Grigoriev A. Analyzing genomes with cumulative skew diagrams. Nucleic Acids Res. 1998;26(10):2286-90

20. Berkhout B, Grigoriev A, Bakker M, Lukashov W. Codon and amino acid usage in retroviral genomes is consistent with virus-specific nucleotide pressure. AIDS Res Hum Retroviruses. 2002;18(2):133-41.

21. Wright F. The 'effective number of codons' used in a gene. Gene. 1990; 87(1):23-9.
22. Bennetzen JL, Hall BD. Codon selection in yeast. J Biol Chem. 1982; 257(6):3026-31.

23. Sharp PM, Li WH. The codon Adaptation Index-a measure of directional synonymous codon usage bias, and its potential applications. Nucleic Acids Res. 1987;15(3):1281-95.

24. Simmonds P. Recombination and selection in the evolution of picornaviruses and other Mammalian positive-stranded RNA viruses. J Virol. 2006;80(22):11124-40

25. Nakamura Y, Gojobori T, Ikemura T. Codon usage tabulated from international DNA sequence databases: status for the year 2000. Nucleic Acids Res. 2000;28(1):292.

26. Faria NR, Azevedo RD, Kraemer MU, Souza R, Cunha MS, Hill SC, et al. Zika virus in the Americas: Early epidemiological and genetic findings. Science. 2016.

27. Musso D, Gubler DJ. Zika Virus. Clin Microbiol Rev. 2016:29(3):487-524.

28. van Hemert FJ, van der Kuyl AC, Berkhout B. The A-nucleotide preference of HIV-1 in the context of its structured RNA genome. RNA Biol. 2013;10(2):211-5.

29. Watts JM, Dang KK, Gorelick RJ, Leonard CW, Bess JWJ, Swanstrom R, Burch $\mathrm{CL}$, Weeks KM. Architecture and secondary structure of an entire HIV-1 RNA genome. Nature. 2009:460(7256):711-6.

30. Vabret N, Bailly-Bechet M, Najburg V, Muller-Trutwin M, Verrier B, Tangy F. The biased nucleotide composition of HIV-1 triggers type I interferon response and correlates with subtype D increased pathogenicity. PLOS One. 2012;7(4):e33502.

31. Kawai T, Akira S. Innate immune recognition of viral infection. Nat Immunol. 2006:7(2):131-7.

32. Girardin SE, Sansonetti PJ, Philpott DJ. Intracellular vs extracellular recognition of pathogens-common concepts in mammals and flies. Trends Microbiol. 2002:10(4):193-9.

33. van Hemert FJ, Berkhout B. The tendency of lentiviral open reading frames to become A-rich: constraints imposed by viral genome organization and cellular tRNA availability. J Mol Evol. 1995;41(2):132-40.

34. Van Hemert F, van der Kuyl AC, Berkhout B. On the nucleotide composition and structure of retroviral RNA genomes. Virus Res. 2014;193:16-23.

35. Berkhout B, van Hemert F. On the biased nucleotide composition of the human coronavirus RNA genome. Virus Res. 2015;202:41-7.

36. Dorn A, Kippenberger S. Clinical application of CpG-, non-CpG-, and antisense oligodeoxynucleotides as immunomodulators. Curr Opin Mol Ther. 2008;10(1):10-20.

37. Karlin S, Doerfler W, Cardon LR. Why is CpG suppressed in the genomes of virtually all small eukaryotic viruses but not in those of large eukaryotic viruses? J Virol. 1994;68:2889-97.

38. Rothberg PG, Wimmer E. Mononucleotide and dinucleotide frequencies, and codon usage in poliovirion RNA. Nucleic Acids Res. 1981;9(23):6221-9.

39. Schubert AM, Putonti C. Evolution of the sequence composition of Flaviviruses. Infect Genet Evol. 2010;10(1):129-36.

40. Lobo FP, Mota BE, Pena SD, Azevedo V, Macedo AM, Tauch A, Machado CR, Franco GR. Virus-host coevolution: common patterns of nucleotide motif usage in Flaviviridae and their hosts. PLoS One. 2009;4(7):e6282

41. Jenkins GM, Holmes EC. The extent of codon usage bias in human RNA viruses and its evolutionary origin. Virus Res. 2003;92(1):1-7.

42. Lara-Ramirez EE, Salazar MI, Lopez-Lopez Mde J, Salas-Benito JS, SanchezVarela A, Guo X. Large-scale genomic analysis of codon usage in dengue virus and evaluation of its phylogenetic dependence. Biomed Res Int. 2014; 2014:851425.

43. Moratorio G, Iriarte A, Moreno P, Musto $\mathrm{H}$, Cristina J. A detailed comparative analysis on the overall codon usage patterns in West Nile virus. Infect Genet Evol. 2013;14:396-400

44. von Lindern JJ, Aroner S, Barrett ND, Wicker JA, Davis CT, Barrett AD. Genome analysis and phylogenetic relationships between east, central and west African isolates of Yellow fever virus. J Gen Virol. 2006:87(Pt 4):895-907.

45. Zhou JH, Zhang J, Sun DJ, Ma Q, Chen HT, Ma LN, Ding YZ, Liu YS. The distribution of synonymous codon choice in the translation initiation region of dengue virus. PLoS One. 2013;8(10):e77239.

46. Behura SK, Severson DW. Nucleotide substitutions in dengue virus serotypes from Asian and American countries: insights into intracodon recombination and purifying selection. BMC Microbiol. 2013:13:37.

47. Zheng H, Shan T, Deng Y, Sun C, Yuan S, Yin Y, Tong G. Molecular characterization of Japanese encephalitis virus strains prevalent in Chinese swine herds. J Vet Sci. 2013;14(1):27-36. 
48. Leifer I, Ruggli N, Blome S. Approaches to define the viral genetic basis of classical swine fever virus virulence. Virology. 2013;438(2):51-5.

49. Jenkins GM, Pagel M, Gould EA, de A Zanotto PM, Holmes EC. Evolution of base composition and codon usage bias in the genus Flavivirus. J Mol Evol. 2001;52(4):383-90.

50. Watterson GA. A stochastic analysis of three viral sequences. Mol Biol Evol. 1992;9(4):666-77.

51. van der Kuyl AC, Berkhout B. The biased nucleotide composition of the HIV genome: a constant factor in a highly variable virus. Retrovirology. 2012;9(1):92.

52. Jenkins GM, Rambaut A, Pybus OG, Holmes EC. Rates of molecular evolution in RNA viruses: a quantitative phylogenetic analysis. J Mol Evol. 2002;54(2):156-65.

53. Kutluay SB, Zang T, Blanco-Melo D, Powell C, Jannain D, Errando M, Bieniasz PD. Global changes in the RNA binding specificity of HIV-1 gag regulate virion genesis. Cell. 2014;159(5):1096-109.

54. de Melo Freire CCI A, de Lima Neto DF, Sall AA, de Andrade Zanotto M. Spread of the pandemic Zika virus lineage is associated with NS1 codon usage adaptation in humans. bioRxiv. 2015.

55. Berkhout B, van Hemert FJ. The unusual nucleotide content of the HIV RNA genome results in a biased amino acid composition of HIV proteins. Nucleic Acids Res. 1994;22(9):1705-11.

56. Haas J, Park E-C, Seed B. Codon usage limitation in the expression of HIV-1 envelope glycoprotein. Curr Biol. 1996;6:315-24.

Submit your next manuscript to BioMed Central and we will help you at every step:

- We accept pre-submission inquiries

- Our selector tool helps you to find the most relevant journal

- We provide round the clock customer support

- Convenient online submission

- Thorough peer review

- Inclusion in PubMed and all major indexing services

- Maximum visibility for your research

Submit your manuscript at www.biomedcentral.com/submit
Biomed Central 